\title{
Neurociências e Educação, o papel da memória na dificuldade de aprendizagem: um estudo sobre as produções nos programas de pós-graduação no Brasil no período de
}

\section{1-2021}

\author{
Neurosciences and Education, the role of memory in learning difficulty: a study on the productions
}

in post-graduation programs in Brazil in the period 2011-2021

Neurociencias y Educación, el papel de la Memoria en la dificultad de aprendizaje: un estudio sobre las producciones em programas de postgrado en Brasil en el período 2011-2021

Recebido: 29/01/2022 | Revisado: 03/02/2022 | Aceito: 09/02/2022 | Publicado: 14/02/2022

\author{
Mariana Leal Tobias dos Reis \\ ORCID: https://orcid.org/0000-0002-9723-7826 \\ Universidade Estadual de Maringá, Brasil \\ E-mail: marianaleal.psicologa@gmail.com \\ Nerli Nonato Ribeiro Mori \\ ORCID: https://orcid.org/0000-0002-6798-5225 \\ Universidade Estadual de Maringá, Brasil \\ E-mail: nnrmori@uem.br
}

\begin{abstract}
Resumo
Dificuldades de memória influenciam o processo de aprendizagem, visto que a capacidade de evocar o conhecimento ao longo do tempo e aplicá-lo é uma necessidade. O Brasil apresentou os piores escores em memória operacional e memória de curto prazo comparado a outras crianças de países como Itália, Inglaterra, Argentina e Canadá. Nesse sentido, a pesquisa buscou verificar como esse tema vem sendo pesquisado no Brasil. Para tanto, realizamos um levantamento dos trabalhos produzidos pelos programas de pós-graduação entre 2011 e 2021 e catalogados pela Biblioteca Digital Brasileira de Teses e Dissertações. Trata-se de uma pesquisa do tipo estado da arte, de cunho bibliográfico e documental e possui caráter descritivo. Após a leitura e análise dos resumos foram selecionadas 12 pesquisas, sendo 2 teses e 10 dissertações, destacando-se 2011 e 2016 como os anos de maior produção, somados $5(41,7 \%)$. Quanto à localização, há um predomínio na região Sudeste $(58,3 \%)$, seguida pelo Sul (25\%) e Nordeste $(16,7 \%)$. Oito (8) dos trabalhos $(66,7 \%)$ abordavam dificuldades relacionadas à memória operacional, e quanto às dificuldades de aprendizagem tratadas estão a leitura e compreensão, dislexia, Transtorno de Déficit de Atenção e discalculia e dificuldade em matemática, perfazendo um total de $8(66,7 \%)$. As informações analisadas evidenciaram a importância da memória para a compreensão das dificuldades de aprendizagem, contudo no período de 10 anos encontrou-se poucas pesquisas que tratavam do assunto, indicando a necessidade de ampliação de estudos, bem como sua associação com neurociências para trilhar estratégias que auxiliem em planos pedagógicos focados em atender necessidades educacionais especiais.
\end{abstract}

Palavras-chave: Dificuldade de memória; Dificuldade de aprendizagem; Educação.

\begin{abstract}
Memory difficulties influence the learning process, since the ability to evoke knowledge over time and apply it is a necessity for students. Brazil had the worst scores in working memory and short-term memory compared to other children from countries such as Italy, England, Argentina and Canada. In this sense, this research aimed to assess how this topic has been researched in Brazil by means of a survey of the works produced by postgraduate programs between 2011 and 2021 and which were catalogued in the database of the Brazilian Digital Library of Theses and Dissertations. This is a state-of-the-art research, of a bibliographic and documentary nature and has a descriptive character. After reading and analyzing the abstracts, 12 studies were selected, being 2 theses and 10 dissertations, highlighting 2011 and 2016 as the years with the highest scientific production, 5 papers total (41.7\%). Regarding location, the Southeast of Brazil (58.3\%) was predominant, followed by the South (25\%) and Northeast (16.7\%). Eight (8) of these papers $(66.7 \%)$ addressed difficulties related to working memory, and among the learning difficulties are reading and comprehension, dyslexia, Attention Deficit Disorder and dyscalculia and difficulty in mathematics, totaling $8(66.7 \%)$. The overall analysis pointed to the importance of memory for understanding learning difficulties, yet in these 10 years few studies were found that dealt with the subject, which shows the need for furthering research, as well as its association with neurosciences so as to trace strategies which may help devise pedagogical plans to meet special educational needs.
\end{abstract}

Keywords: Memory difficulty; Learning disability; Education. 


\begin{abstract}
Resumen
Las dificultades de memoria influyen en el proceso de aprendizaje, ya que la capacidad de evocar conocimientos a lo largo del tiempo y aplicarlos es una necesidad. Brasil obtuvo los peores puntajes en memoria de trabajo y memoria a corto plazo en comparación con países como Italia, Inglaterra, Argentina y Canadá. En ese sentido, la investigación buscó verificar cómo este tema ha sido investigado en Brasil por medio de un levantamiento de los trabajos producidos por los programas de posgrado entre 2011 y 2021 y catalogados en la Biblioteca Digital Brasileña de Tesis y Disertaciones. Se trata de una investigación de vanguardia, de carácter bibliográfico, documental y descriptivo. Luego de la lectura y análisis de los resúmenes, se seleccionaron 12 estudios, siendo 2 tesis y 10 disertaciones, destacándose 2011 y 2016 como los años de mayor producción, sumados 5 (41,7\%). En cuanto a la ubicación, hay predominio en lo Sudeste (58,3\%), seguido por lo Sur (25\%) y Nordeste (16,7\%). Ocho (8) de estos trabajos $(66,7 \%)$ abordaron dificultades relacionadas con la memoria de trabajo, y entre las dificultades se encuentran la lectura y comprensión, dislexia, Trastorno por Déficit de Atención y discalculia y dificultad en matemáticas. La análisis mostró la importancia de la memoria para la comprensión de las dificultades de aprendizaje, sin embargo, in este período se encontraron pocos estudios que trataran el tema, mostrando la necesidad de incentivar los estudios y asociarlos con neurociencias para trazar estrategias que ayuden en los planes pedagógicos para atender las necesidades educativas especiales.
\end{abstract}

Palabras clave: Dificultades de memoria; Dificultades de aprendizaje; Educación.

\title{
1. Introdução
}

A educação tem por finalidade o desenvolvimento de novos conhecimentos ou comportamentos, sendo mediada por um processo que envolve a aprendizagem. Aprendemos quando somos capazes de transformar nossas práticas e o mundo em que vivemos, nos tornando pessoas que vivem em sociedade (Cosenza et al., 2011).

Nessa perspectiva, a aprendizagem pode ser definida como alterações cerebrais resultantes de experiência (Sant'Ana, 2015). Em outros termos, pode ser compreendida como o processo de aquisição de informação (Gazzaniga, 2006), sendo o cérebro humano o principal envolvido nesse processo, sendo responsável por atividades complexas, como o pensamento, a memória, a emoção e a linguagem (Aamodt et al., 2013).

As neurociências descrevem a estrutura e funcionamento do sistema nervoso, como os neurônios e suas moléculas constituintes, os órgãos e funções específicas do sistema nervoso.

$\mathrm{Na}$ educação, a neurociência tem destaque no documento publicado pela Organização de Cooperação e Desenvolvimento Econômico (OCDE, 2003), apontando novas perspectivas sobre a aprendizagem com base em pesquisas sobre o cérebro.

Desta forma, esta ciência tangenciada pela educação nos processos de aprendizagem, pode proporcionar estratégias pedagógicas de ensino-aprendizagem, de modo a oferecer estímulos que favoreçam a reorganização do sistema nervoso em desenvolvimento, resultando em maiores mudanças comportamentais (Guerra, 2011).

O diálogo entre neurociências e educação, pode proporcionar que professores atuem como agentes nas mudanças cerebrais que levam à aprendizagem, pois enquanto a educação cria condições que promovem o desenvolvimento de competências, os professores podem favorecer a um aprendizado mais eficiente e significativo por conhecer o funcionamento cerebral (Cosenza et al., 2011). De acordo Bortoli e Teruya (2017, p. 70) "os estudos da neurociência no campo da educação são uma alternativa para repensar as práticas pedagógicas na contemporaneidade".

A memória é uma das funções cognitivas muito importante envolvida na aprendizagem, sendo que esta não é um sistema unitário e, de maneira geral, consiste na capacidade de reter e usar informações e conhecimentos, e corresponde ao meio que usamos para evocar experiências passadas e usar as informações no presente (Barbosa et al., 2016).

Processos mnemônicos significam aquisição, formação, conservação e evocação de informações. A aquisição é também chamada de aprendizado ou aprendizagem: só se "grava” aquilo que foi aprendido. A evocação é também chamada de recordação, lembrança, recuperação. Só lembramos aquilo que gravamos, aquilo que foi aprendido (Consenza et al., 2011). 
O acervo das memórias de cada um nos converte em indivíduos, utilizamos, a partir dos 2 ou 3 anos, a linguagem para adquirir, codificar, guardar ou evocar memórias; as demais espécies animais, não. Mas, fora as áreas da linguagem, usamos mais ou menos as mesmas regiões do cérebro e mecanismos moleculares semelhantes em cada uma delas para construir e evocar memórias totalmente diferentes (Izquierdo, 2014).

Dificuldades de memória influenciam o processo de aprendizagem, pois para se ter um aprendizado efetivo é necessário ser capaz de evocar o conhecimento ao longo do tempo e ainda aplicá-lo de diferentes formas. De acordo com o estudo de Alloway et al. (2017), o Brasil apresentou os piores escores em memória operacional e memória de curto prazo comparado à outras crianças de países como Itália, Inglaterra, Argentina e Canadá. Segundo o autor, estes dados podem estar associados fato de apresentarmos queda nos índices relacionados à qualidade de ensino e a discrepância entre o ranking educacional.

O presente estudo tem como objetivo verificar como o tema vem sendo estudado no Brasil, entendendo sua grande relevância para a educação. Para tanto, foi realizado um levantamento e análise dos trabalhos produzidos pelos programas de pós-graduação brasileiros no período de 2011 a 2021 e catalogados no banco de dados da Biblioteca Digital Brasileira de Teses e Dissertações (BDTD, 2021).

Para apresentar o percurso metodológico e os resultados alcançados em nossa pesquisa, iniciamos com a fundamentação teórica, abordando os aspectos neurobiológicos da memória e aprendizagem, e na sequência, focamos nos procedimentos metodológicos para a coleta e análise de dados.

\section{Metodologia}

A pesquisa pode ser caracterizada como quali-quantitativa por envolver o que Pereira, Shitsuka, Parreira e Shitsuka (2018) e Severino (2016) definem como discussão analítica de dados quantitativos. De modo mais específico, trata-se de uma pesquisa denominada estado da arte, visto que está voltada para o mapeamento e análise de um conhecimento determinado em um espaço de tempo. Este estudo, utilizou-se como modelo a pesquisa realiza por Mori et al (2021), no qual realizaram uma investigação sobre as produções nos programas de pós-graduação no Brasil no período de 2002-2020, mas como foco o tema altas habilidades/superdotação.

Para Ferreira (2002), o estado da arte tem um caráter bibliográfico, mas avança para além da revisão bibliográfica, pois busca aspectos, dimensões e condições de produção de uma temática em textos acadêmicos diversos. Para a autora, é um proceder metodológico de levantamento, avaliação e síntese de estudos já realizados sobre um assunto.

Segundo Norma (2021) estudos denominados estados da arte, principalmente no campo da Educação colaboram para o conhecimento e análise social a cerca de um tema, o que contribue para a implementação de políticas públicas que favorecessem o desenvolvimento do país.

A Biblioteca Digital Brasileira de Teses e Dissertações (BDTD) é um dos sistemas que faz parte do Programa Brasileiro de Acesso Aberto à Informação Científica, coordenado pelo Instituto Brasileiro de Informação em Ciência e Tecnologia (IBICT). A BDTD foi o primeiro sistema a utilizar os conceitos de Arquivos Abertos, sendo assim, modelo para o desenvolvimento de outros produtos/serviços.

\section{Breve Explicação Neurobiológica da Memória e Aprendizagem}

As memórias são feitas por células nervosas (neurônios), se armazenam em redes de neurônios e são evocadas pelas mesmas redes neuronais ou por outras. O processo de ativação dos sistemas mnemônicos surge quando um evento qualquer, seja ele externo ou interno, ativa alguma região neural. Os eventos externos são mais acessíveis aos métodos 
objetivos da neurociência. Os eventos internos podem ser os nossos próprios pensamentos e sentimentos, ou então, mais recentemente, por meio das técnicas de imagem funcional e registro eletrofisiológico não invasivo (Lent, 2010).

As emoções são importantes moduladores da memória, pelo nível de consciência e pelos estados de ânimo. Desta forma, é mais fácil aprender ou evocar algo quando estamos alertas e de bom ânimo; e como fica difícil aprender qualquer coisa, ou até lembrar quando deprimidos ou muito estressados. Assim a emoções, o contexto e a combinação de ambos influenciam a aquisição e a evocação (Izquierdo, 2014).

Os estudos sobre o cérebro apontam que as emoções contribuem para a aprendizagem porque auxiliam a passagem da memória de curto para a memória de longo prazo, assim como a motivação é indispensável para a liberação de substância responsável por mobilizar a atenção e reforçá-la na relação com o objeto que a afetou (Bortoli \& Teruya, 2017, p. 70).

A memória pode ser classificada com relação ao tempo de retenção e à sua natureza. Quanto ao tempo de retenção em. (1) memória ultrarrápida ou imediata, cuja retenção não dura mais que alguns segundos; (2) memória de curta duração, que dura minutos ou horas e serve para proporcionar a continuidade do nosso sentido do presente, e (3) memória de longa duração, que estabelece recordações mais duradouras (dias, semanas e até mesmo anos) (Lent, 2010).

E quanto à sua natureza em: (1) memória explícita ou declarativa; (2) memória implícita ou não declarativa e (3) memória operacional ou memória de trabalho. A memória explícita reúne tudo que só podemos evocar por meio de palavras, pode ser episódica, quando envolve eventos datados, isto é, relacionados ao tempo; ou semântica quando envolve conceitos atemporais (Lent, 2010).

A memória episódica é geralmente específica de cada indivíduo, característica de sua trajetória de vida. A memória semântica, por outro lado, é compartilhada por muitas pessoas, fazendo parte da cultura (Lent, 2010).

A memória implícita, por sua vez, é diferente da explícita porque não precisa ser descrita com palavras. Além disso, requer mais tempo e treinamento para se formar, mas persiste mais duradouramente, esta fortemente ligada a memória de procedimentos, hábitos e habilidades e das regras em geral, como por exemplo dirigir um carro (Lent, 2010).

Através da memória operacional, armazenamos temporariamente informações que serão úteis apenas para o raciocínio imediato e a resolução de problemas, ou para a elaboração de comportamentos, podendo ser descartadas (esquecidas) logo a seguir. Contudo, este tipo de memória é muito importante para o aprendizado, pois permite manipular a informação aprendida, a após ser selecionada a relevância da informação esta pode ser dirigida para uma memória de longo prazo (Lent, 2010; Guerra, 2011).

A memória operacional é a capacidade para reter e manipular informações por curtos períodos - possui modalidades visuais e verbais - e está associada, entre outros, à resolução de problemas. Esta habilidade também é responsável pelo resgate das informações junto à memória de longo prazo (Baddeley, 1986).

A memória é formada por uma série de sistemas diferentes que têm em comum a capacidade de armazenar informações e retê-las por determinado tempo, podendo ser desde frações de segundos até a vida inteira (Baddeley, 1999).

Sob esta perspectiva, a memória e a aprendizagem envolvem processos cognitivos estreitamente relacionados, que causam mudanças adaptativas no comportamento dos organismos por meio de experiências adquiridas e armazenadas (Bernal, 2005). Da mesma forma, que este processo de aquisição das novas informações, aprendizagem, viabilizam os processos neurobiológicos e neuropsicológicos da memória (Lent, 2010; Izquierdo, 2014).

A partir de um estímulo visual, a imagem penetra pela retina, e é transformada em sinais elétricos, chega através de várias conexões neuronais ao córtex occipital e lá causa uma série de processos bioquímicos. Um estímulo externo, verbal, 
embora possa penetrar também pela retina (por exemplo, quando lemos), acaba em outras regiões do córtex cerebral (Izquierdo, 2014).

No cérebro há regiões do em que todas essas vias convergem, sendo pontos usados na formação e na evocação de memórias (Izquierdo, 2014). A consolidação da memória envolve o fortalecimento das associações entre as novas memórias que chegam (provenientes dos sistemas mnemónicos de curta duração) e a informação previamente existente, um processo que pode durar alguns anos no homem (Lent,2010).

Ao converter a realidade num complexo código de sinais elétricos e bioquímicos, os neurônios traduzem, e na evocação reconvertem sinais bioquímicos ou estruturais em elétricos, de maneira que novamente nossos sentidos e nossa consciência possam interpretá-los como pertencendo a um mundo real. Em cada tradução ocorrem perdas ou mudanças (Izquierdo, 2014).

A informação passa pelo filtro da atenção, em seguida provoca a ativação de neurônios caracterizando a memória operacional, se essa experiência de aprendizado for relevante vai provocar alterações estruturais em circuitos nervosos, promovendo um registro, que para se tornar um traço permanente necessita passar por processos de repetição, elaboração e consolidação (Consenza et al., 2011).

Quantos mais complexos forem os processos envolvidos mais forte será esse registro, e assim a consolidação provocará alterações biológicas nas ligações entre neurônios, fazendo com esse registro se vincule a outros já existentes, tornando-se permanentes (Consenza et al., 2011).

O hipocampo não é o sítio onde estão armazenadas as memórias explícitas, mas a estrutura coordenadora do processo de consolidação, que provavelmente se realiza em outros setores do córtex. Essa hipótese é apoiada pelas abundantes conexões que o hipocampo possui com as demais regiões do lobo temporal medial, e através destas com diversas regiões corticais, especialmente o córtex pré-frontal, o córtex parietal e as regiões anteriores e laterais do lobo temporal (Lent, 2010).

\section{Dificuldade de Memória e de Aprendizagem nas Teses e Dissertações}

A pesquisa tem como foco os estudos sobre dificuldades de memória e de aprendizagem nos programas de pósgraduação brasileiros. Para tanto, realizamos um levantamento dos trabalhos produzidos pelos programas de pós-graduação entre 2011 e 2021 e catalogados no banco de dados da Biblioteca Digital Brasileira de Teses e Dissertações (BDTD, 2021), vinculada ao Instituto Brasileiro de Informação em Ciência e Tecnologia, do Ministério da Ciência, Tecnologia e Inovação.

Criada em 2002, a BDTD é mantida pelo Instituto Brasileiro de Informações em Ciências Tecnologia - IBICT, com o apoio de Financiadora de Estudos e Pesquisas (FINEP). A plataforma armazena e disponibiliza, de modo eletrônico, teses e dissertações realizadas na pós-graduação.

Para o levantamento utilizamos os termos dificuldade de memória e dificuldade de aprendizagem, o qual retornou um total de 190 trabalhos, sendo 62 teses e 128 dissertações. Com a leitura dos resumos, constatamos que alguns trabalhos não tratavam do assunto e por isso, eles foram descartados, e o resultado está demonstrado no Quadro 1.

Quadro 1: Teses e dissertações sobre dificuldade de memória e dificuldade de aprendizagem - 2011 a 2021.

\begin{tabular}{|l|l|l|}
\hline Nível & Resultado Inicial & Após leitura dos resumos \\
\hline Dissertação & 128 & 10 \\
\hline Tese & 62 & 2 \\
\hline Total & 190 & 12 \\
\hline
\end{tabular}

Fonte: Autores. 
Como podemos ver no Quadro 1, após a leitura inicial, o conjunto a ser analisado ficou constituído por 12 textos, sendo 10 dissertações $(83,3 \%)$ e 2 teses (16,6\%) defendidas em 9 instituições. Na continuidade, os dados foram organizados conforme as seguintes categorias temáticas: distribuição dos trabalhos por nível e ano, instituição de origem e localização geográfica e assuntos tratados.

Quanto à distribuição por nível e ano, os dados indicam que os anos de maior quantidade de pesquisas foi nos anos de 2011 e 2016, sendo no total de três trabalhos, no no restante dos anos apenas uma; após esse período não há registros de pesquisas sobre o tema.

Os estudos sobre neurociências e o funcionamento do cérebro tiveram maior destaque a partir do ano 2000, (Herculano-Houzel, 2002), (Kandel, 2003), (Gazzaniga, 2006), (Lent, 2010), (Guerra, 2011), o que não corroborou proporcionalmente com mais estudos envolvendo dificuldade de memória e dificuldade de aprendizagem, evidenciando a necessidade de pesquisas nesta área por se tratar de um tema de grande relevância no que se refere aos processos de aprendizagem.

Ao averiguar onde foram produzidos os estudos, podemos ver na Figura 1 que a maior parte deles está concentrada em instituições da região Sudeste (58,3\%), seguida pelas regiões Sul (25\%) e Nordeste $(16,7 \%)$, as demais regiões não tiveram nenhum estudo relacionado ao assunto neste período.

Figura 1: Pesquisa sobre dificuldade de memória e de aprendizagem por região.

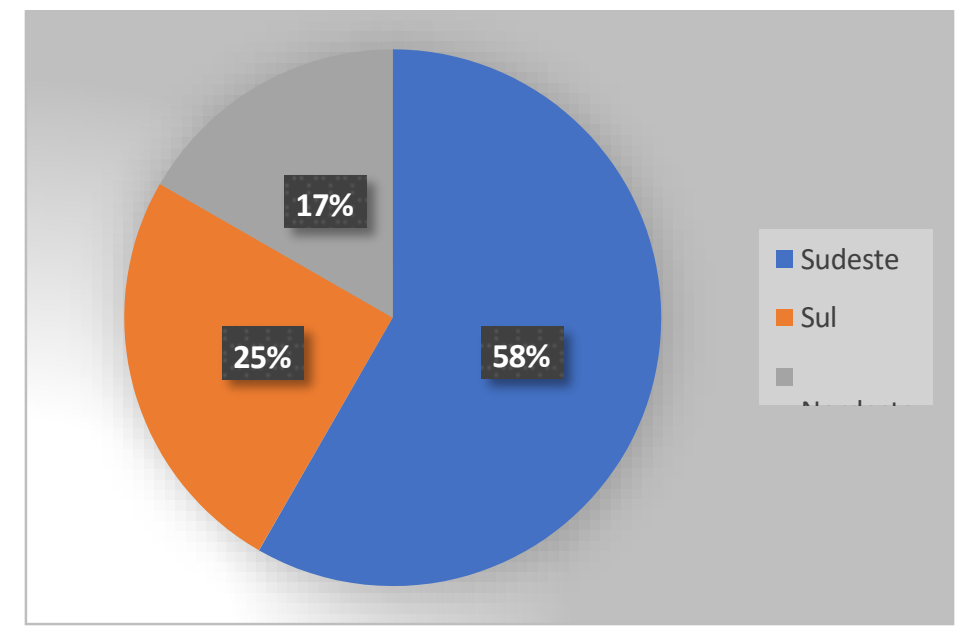

Fonte: Autores.

As instituições com mais trabalhos defendidos sobre o tema estão localizadas na Universidade Estadual Paulista (UNESP) e na Universidade Federal do Rio Grande do Sul (UFRS), que somam juntas $(5,41,6 \%)$ do total das produções. As demais produções estão localizadas na Universidade de São Paulo (USP, 1), Universidade Federal de São Paulo (UNIFESP, 1), Universidade Estadual de Campinas (UNICAMP, 1), Universidade Estadual de Londrina (UEL, 1), Universidade Federal do Minas Gerais (UFMG, 1), Universidade Federal da Paraíba (UFPA, 1), Universidade Federal do Rio Grande do Norte (UFRN, 1).

Com relação aos assuntos trabalhados $(8,66,7 \%)$ abordavam dificuldades relacionadas a memória trabalho ou operacional, e (4, 33,3\%), conforme figura 2, descreviam dificuldades de outros tipos de memória, ou tratavam do assunto de uma forma mais completa. De acordo com Baddeley (1986), a memória operacional desempenha um papel crucial em muitas formas de cognição complexa tais como a aprendizagem, o raciocínio e a compreensão da linguagem falada, escrita, da 
aritmética mental e resolução de problemas. E desta forma, falhas neste sistema podem provocar prejuízos no processo de aprendizagem, na leitura e compreensão de um texto, e na resolução de problemas de matemática.

Segundo estudo de Alloway (2010), o mau funcionamento de um ou mais componentes da memória operacional está relacionado com as dificuldades de aprendizagem e ao baixo rendimento escolar, o que se justifica por apresentar maior quantidade de estudos envolvendo esta área. Contudo, o funcionamento do cérebro e da aprendizagem é um sistema complexo, influenciado por diversos elementos, sugerindo que para se desenvolver estratégias efetivas de aprendizado baseado em neurociências outros componentes cognitivos necessitam também serem estudados por meio de pesquisas mais completas relacionando a memória e as dificuldades de aprender.

Figura 2: Assuntos abordados nas pesquisas.

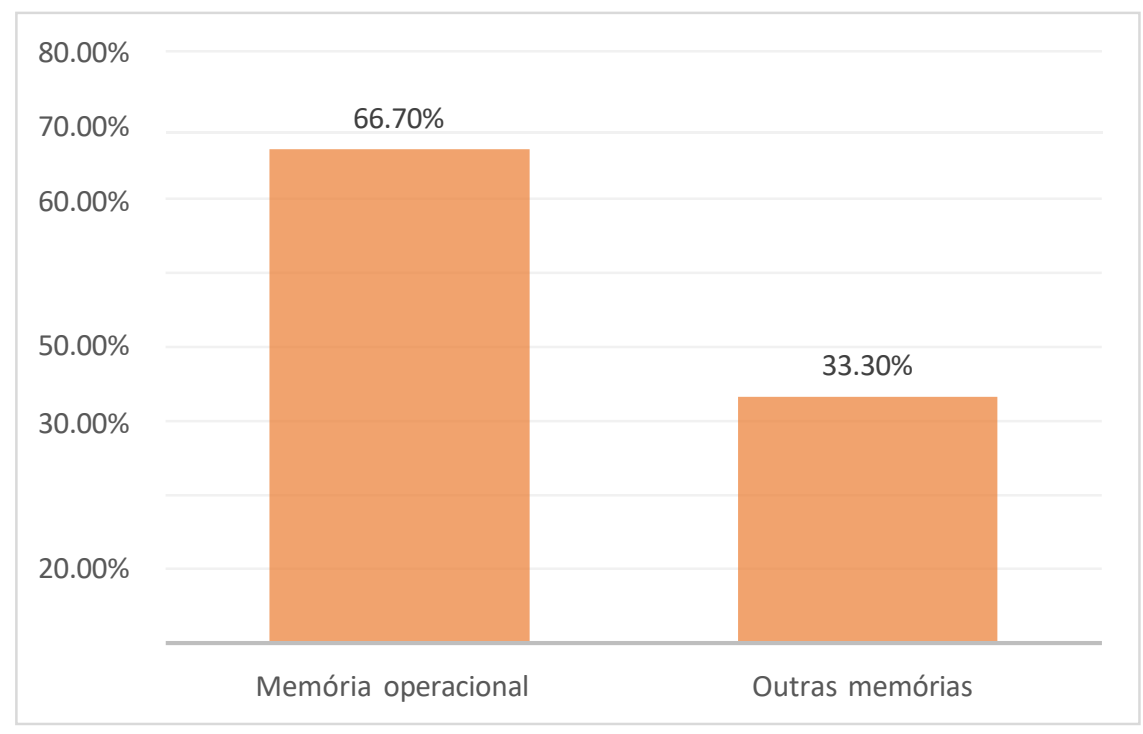

Fonte: Autores.

Com relação às dificuldades de aprendizagem estudadas estão leitura e compreensão, dislexia, Transtorno de Déficit de Atenção e discalculia e dificuldade em matemática, perfazendo um total de $(8,66,7 \%)$, no restante dos trabalhos $(2$, $16,7 \%)$ se referiam a memória operacional fonológica e dificuldades associadas, $(1,8,3 \%)$ sobre memória de procedimento e gagueira e $(1,8,3 \%)$, memória operacional e dificuldade de aprendizagem em geral.

A compreensão das dificuldades de memória e sua relação com as dificuldades de aprendizagem qualquer que seja ela, é capaz de promover estratégias mais eficazes para superá-lo assim como orientar pais e professores a mediarem de forma precoce estimulando este recurso cognitivo tão efetivo.

A ampliação do debate sobre o tema proporcionará maior interesse possibilitando aumentar o volume das pesquisas e publicações, e consequente maior acesso aos profissionais de educação, às famílias dos alunos e comunidade em geral. Oliveira (2021) afirma que a compreensão do papel crucial da memória no processo de aprendizagem escolar promove benefícios da aplicação pedagógica. E, dessa forma, poderão delinear estratégias pedagógicas baseadas em neurociências que visem estimular o cérebro e superar as dificuldades de aprendizagem. 


\section{Considerações Finais}

Os dados apresentados e discutidos são frutos de uma pesquisada realizada com o intuito de verificar como o tópico dificuldade de memória e dificuldade de aprendizagem vem sendo estudados em programas de pós-graduação brasileiros no período de 2011 a 2021. Os dados foram colhidos nos trabalhos catalogados no banco de dados da Biblioteca Digital Brasileira de Teses e Dissertações.

As informações analisadas mediante a leitura dos trabalhos evidenciaram a importância da memória para compreensão das dificuldades de aprendizagem, no entanto no período de 10 anos encontrou-se poucas pesquisas que tratavam do assunto indicando a necessidade de ampliação de estudos sobre especificidades relacionadas à dificuldade de memória e dificuldades de aprendizagem, bem como a sua associação com neurociências com o objetivo de trilhar estratégias que auxiliem em plano pedagógicos para atender necessidades educacionais especiais.

Entende-se que a compreensão deste tema, possibilita a transposição educacional destes conhecimentos e aplicação de experiências e oportunidades pedagógicas na promoção do desenvolvimento, contudo sugere-se que mais pesquisas sejam realizadas na área e divulgadas em veículos e contextos acessíveis aos professores e demais profissionais relacionados com o contexto escolar, bem como comunidade em geral.

\section{Referências}

Alloway, T. P., \& Alloway, R. G. (2010). Investigating the predictive roles of working memory and IQ in academic attainment. Journal of Experimental Child Psychology, 106 (1), 20-9.

Baddeley, A. D. (1986). Working memory. Oxford University Press.

Baddeley, A. D. (1999). Essentials of human memory. Psychology Press.

BDTD. (2021). Biblioteca digital de teses e dissertações. https://bdtd.ibict.br/vufind/.

Bernal, M. I. (2005). Psicobíologia del aprendizage y la memoria. CIC - Cuadernos de Informácion y Comunicátion, Norteamérica, 10, $221-33$.

Bortoli, B., \& Teruya, T. K. (2017). Neurociência e Educação: Os Percalços e possibilidades de um caminho em construção. Imagens da Educação, 7(1), 70-7.

Cosenza, R., \& Guerra, L. B. (2011). Neurociência e educação: como o cérebro aprende. Artmed.

Ferreira, N. S. (2002). As pesquisas denominadas "estados da arte". Educação e Sociedade, ano XXIII, $23(79), 257-72$.

Ferreira, N. S. (2021). Pesquisas Intituladas Estado da Arte: Em foco. Rev. Int. de Pesq. em Didática das Ciências e Matemática (RevIn), 2. Gazzaniga, M., Mangun, G. R. \& Ivry, R. B. (2006). Neurociência cognitiva: a biologia da mente. Artmed.

Guerra, L. B. (2011). O diálogo entre a neurociência e a educação: da euforia aos desafios e possibilidades. Revista Interlocução, 4(4), 01-10.

Herculano-Houzel, S. (2002). O cérebro nosso de cada dia. Casa Editorial.

Izquierdo, I. (2011). Memória. Artmed.

Kandel, E. (2003). O sistema nervoso e o comportamento. In : E. Kandel, J. Schwartz, \& T. M. Jessel (Orgs.). Princípios da neurociência (pp. 13-37). Manole.

Lent, R. (2010). Cem bilhões de neurônios? Conceitos fundamentais de neurociência. Atheneu.

Mori, N. N. R.., Saito, D., Risso, V. A. M. \& Macias, V. M. (2021). Altas habilidades/superdotação na pesquisa brasileira: um estudo sobre as produções nos programas de pós-graduação no Brasil no período de 2002-2020. Research, Society and Development, 10 (2).

Oliveira, C. (2021). O papel da memória humana na aprendizagem escolar e na inovação pedagógica. In: Políticas e Dinâmicas Educativas (pp. 29-45). Coimbra.

Pereira, A. S., Shitsuka, D. M., Parreira, F. J. \& Shitsuka, R. (2018). Metodologia da pesquisa científica UFSM. https://repositorio.ufsm.br/bitstream/handle/1/15824/Lic_Computacao_Metodologia-Pesquisa-Cientifica.pdf?sequence=1.

Sant'Ana, D. M. G. (2015). Plasticidade neural: as bases biológicas da aprendizagem. In: C. L. Chitolina, J. A. Pereira, \& R. H. Pinto (Orgs.). Mente, cérebro e consciência: um confronto entre a filosofia e ciência (vol. 1, pp. 73-84). Paco Editorial.

Severino, A. J. (2016). Metodologia do trabalho científico. Cortez. 